\title{
38-year-old woman with recurrent abdominal pain, but no fever
}

\author{
This article was published in the following Dove Press journal: \\ International Journal of General Medicine \\ 21 March 2012 \\ Number of times this article has been viewed
}

\section{Kentaro Iwata' \\ Tomoko Toma ${ }^{2}$ \\ Akihiro Yachie ${ }^{2}$ \\ 'Department of Infectious Diseases, Kobe University Hospital, Kobe, Japan; ${ }^{2}$ Department of Pediatrics, Graduate School of Medical Science and School of Medicine, Kanazawa University, Kanazawa, Japan}

\begin{abstract}
A 38-year-old woman presented with 2 days history of left-flank pain. She had similar episodes of abdominal pain as well as chest pain several times, but symptoms disappeared spontaneously. Each time she developed pain, there was no fever. After ruling out common causes of recurrent abdominal pain, familial Mediterranean fever (FMF) was considered as a potential diagnosis. Genetic tests revealed multiple heterozygote mutations, which may be associated with FMF. Patients with Mediterranean fever mutations may present with atypical presentations without fever, like in this case. Astute clinical suspicion is required to make an accurate diagnosis.
\end{abstract}

Keywords: familial Mediterranean fever, MEFV mutation, afebrile

\section{Introduction}

Abdominal pain in premenopausal women is often a diagnostic challenge. We describe a case of 38-year-old woman who presented with recurrent abdominal pain. A conventional work-up failed to reveal a diagnosis. A detailed history suggested recurrent serositis syndrome. Although the patient denied fever, a diagnosis of FMF was considered. We discuss the rationale for the diagnosis and interpretation of genetic tests.

\section{Case report}

A 38-year-old woman presented reporting 2 days of left-sided abdominal pain. The pain was intermittent in nature. She denied any fever, gastrointestinal symptoms, respiratory symptoms and genitourinary symptoms. Her last menstrual period was a few weeks ago and she denied pregnancy. Eight years prior to the current presentation, she developed left-side chest pain worsened by deep inspiration. A chest radiograph reportedly revealed atelectasis and she was diagnosed with "pleuritis with middle lobe lingual syndrome". She did not recall whether she had a fever since then. Three years ( 44 months to be precise) prior to the current presentation, she developed acute onset left-flank pain without fever. She visited a urology clinic but the urinalysis was normal. An urologist who saw the patient considered her condition was unlikely to be urolithiasis but was not able to find an alternative explanation. Her pain disappeared without specific treatment several days later. Thirty-five months prior to the current presentation, she developed left-side chest and back pain without fever. Blood tests showed a white blood cell (WBC) count of 7,000/. $\mathrm{mm}^{3}$ and C-reactive protein (CRP) of $1.69 \mathrm{mg} / \mathrm{dL}$. There was an elevated liver function test (aspartate aminotransferase
Correspondence: Kentaro Iwata Kusunokicho 7-5-I, Chuoku, Kobe, Hyogo 650-0017, Japan Email kiwata@med.kobe-u.ac.jp 
[AST] $48 \mathrm{U} / \mathrm{L}$, alanine aminotransferase [ALT] $66 \mathrm{U} / \mathrm{L}$ ). A physician caring for her diagnosed it as pleuritis and an oral antibiotic was prescribed. At a follow-up visit 3 days later, the patient became asymptomatic and laboratory abnormalities were all normalized. Twenty-six months prior to the current presentation, she developed another left-side chest pain without fever. A laboratory exam revealed WBC $7600 / \mathrm{mm}^{3}$ and CRP was $<0.24 \mathrm{mg} / \mathrm{dL}$. She was again diagnosed with pleuritis, and symptoms improved several days later (treatment detail unknown). Twenty-five months prior to the current presentation, she developed another abdominal pain. Pain was associated with her position and movement. WBC was $8600 / \mathrm{mm}^{3}$, with neutrophil $67 \%$ (4\% band form), lymphocyte $27 \%$, CRP $1.32 \mathrm{mg} / \mathrm{dL}$, and erythrocyte sedimentation rate (ESR) $36 \mathrm{~mm} /$ hour. The abdominal ultrasound was normal and a computed tomography scan without contrast of abdomen revealed incidental findings of spina bifida at sacrum and fatty liver but no abnormality at urinary tract or elsewhere. She had had no episode of pain since then until this current presentation.

Her past medical history was significant only for childhood inguinal hernia, which was repaired surgically, and freckles, which were treated with topical agents. She denied allergy to medications, took no alcohol or illicit drugs, and denied tobacco smoking. She became pregnant twice and was the mother of 12- and 14-year-old children. There was no significant family history. She only took overthe-counter vitamins.

Upon presentation, the patient appeared well but suddenly started to suffer abdominal pain during the interview. She was not able to hold herself upright and had to lie down on a bed. On physical examination, she appeared ill. Her blood pressure was $125 / 76 \mathrm{mmHg}$, pulse rate 84 /minute, respiratory rate 22 /minute, and body temperature taken at axilla was $36.8^{\circ} \mathrm{C}$. Her pain worsened upon deep inspiration. However, her physical examination was otherwise unremarkable, and there was no tenderness on her abdomen or on her back. Laboratory tests showed a CRP of $1.45 \mathrm{mg} / \mathrm{dL}$ (reference range $<0.24 \mathrm{mg} / \mathrm{dL}$ ) and an ESR of $25 \mathrm{~mm}$ at 1 hour. The WBC differential was neutrophil $65.0 \%$ (5\% band form), lymphocyte $22.0 \%$, monocyte $13.0 \%$, and eosinophil $0 \%$. Due to a technical error, $\mathrm{CBC}$ was not performed, but later in the day it turned out to be normal. Electrolytes, liver function tests, and kidney function tests were all normal. Urinalysis was negative for protein and sugar, but there was 10-19 RBC/HPF, 30-49 WBC/HPF, with trace bacteria. Serum thyroid-stimulating hormone (TSH) level was normal.
8 years PTCP: Left-side chest pain with pleuritis?

$$
\downarrow
$$

44 months PTCP: Left-flank pain. Urolithiasis?

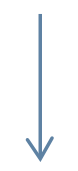

35 months PTCP: $\quad$ Left-side chest pain, back pain and abnormal liver function

26 months PTCP: Left-side chest pain

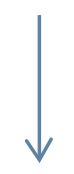

25 months PTCP: Abdominal pain

\section{Current presentation: Left-flank pain}

Figure I Patient's history of recurrent pain, prior to current presentation (PTCP).

Her cause of abdominal pain was judged to be serositis, based on history and physical examination. After resting, her pain gradually improved and she was sent home with prescription of non-steroidal anti-inflammatory medicine as needed. On the follow-up visit a week later, she was completely asymptomatic. Rheumatoid factor, anti-nuclear antibody (ANA), complement level, C3, C4 level, and serum ferritin level measured at the first visit all turned out normal. Urinary lead and porphobilinogen were negative. Given the recurrent nature of multiorgan serositis, FMF was considered as a diagnosis despite the lack of fever. A genetic blood test was performed at the Department of Pediatrics, Graduate School of Medical Science and School of Medicine, Kanazawa University, and it revealed $M E F V$ mutations at exon 3 (P369S and R408Q) and intron 8 (IVS8+8 C-T). The symptom has not recurred since this interview. After a discussion with the patient, prophylactic colchicine was not prescribed, and genetic tests for family members were not performed. 


\section{Discussion}

Abdominal pain in premenopausal women can be a diagnostic challenge. Its cause may range from gastrointestinal, hepatobiliary, or urological to obstetric and gynecological. Occasionally, some diseases cause abdominal pain without findings on imaging studies, such as diabetic ketoacidosis, acute intermittent porphyria, lead poisoning, or conversion disorder. However, detailed history taking and physical examination often lead to accurate diagnosis. Moreover, the list of differential diagnoses of left-side abdominal pain, as in our case, is not long, compared to right-side abdominal pain.

FMF is an autosomal recessive, inherited periodic inflammatory syndrome, characterized by self-limited recurrent attacks of fever with serositis such as peritonitis, pleuritis and arthritis. ${ }^{1}$ It is known to occur mainly among Mediterranean and Middle Eastern populations such as non-Ashkenazi Jews, Arabs, Turks, and Armenians. Despite its name, FMF is now shown to occur worldwide. In Japan, more than 90 cases of FMF were reported..$^{2-4}$ Its onset is usually early but onset over 50 years old has been reported. ${ }^{5}$ High fever is present in more than $90 \%$ of cases with FMF, ${ }^{6}$ and fever was present in $98.8 \%$ of cases in a Japanese study. ${ }^{3}$ However, this may be due to underdiagnoses and underreporting of afebrile patients. In fact, the current diagnostic criteria do not necessarily require fever for the diagnosis. ${ }^{1}$ This patient fulfilled only one minor criterion on the simplified version of the criteria, which is now widely used. However she fulfilled two minor and five supportive criteria of the more detailed version; ie, incomplete attacks of abdomen and chest (minor criteria), and severe attacks requiring bed rest, spontaneous remission, symptom-free interval, transient inflammatory response, and episodic hematuria (supportive criteria), for which the specificity of the diagnosis is $99 \% .{ }^{1}$

Patients with FMF at late onset tend to have a less severe form, and this might in part explain her lack of fever. In fact, the Tel Hashomer Severity Score of the patient was only ${ }^{1,7}$ which includes age in the scoring, suggesting she had a milder form of FMF.

FMF is caused by mutations in the Mediterranean fever gene $(M E F V)$ on chromosome 16p13.3, which encodes protein named pyrin. Pyrin regulates production of IL-1 $\beta$ and activation of NF- $\mathrm{\kappa B}$. Lack of normal pyrin activity in FMF is considered to be the cause of excess cytokines and subsequent inflammatory attack. ${ }^{8}$

Diagnosis of FMF is usually made clinically. Genetic tests are not necessarily mandated, and the results may be normal in patients with FMF. Likewise, a family history may not be detected, as in our case. $M E F V$ mutations commonly seen in Japan are different from those of Mediterranean people, where M694V is most common. Typical mutations in Japanese patients are E148Q on exon 2, and M694I on exon $10 .{ }^{3,8}$ Our patient did not have either, and it may partly explain the milder form of symptoms. A combination of P369S and R408Q, which was detected in our case, was found in three Japanese cases, but there was only one case without E148Q. ${ }^{3}$ P369S and R408Q can be found even in healthy Japanese subjects. In one study, $3.9 \%$ and $3.3 \%$ of healthy Japanese had P369S and R408Q mutations, respectively. ${ }^{9}$ According to The Registry of Hereditary Auto-Inflammatory Disorders Mutations database (http://fmf.igh.cnrs.fr/ISSAID/ infevers/), the combination of P369S and R408Q was first detected in an Armenian patient with FMF in 2007. Also, IVS8+8 C-T was detected together with L110P, E148Q, P369S, R408Q, and R501R in a Vietnamese patient. However, a diagnosis of FMF was clinically not clear in the latter. The precise role of these mutations is not known, and they are seen in cases ranging from full-blown FMF to completely asymptomatic persons. ${ }^{10}$ In addition, these genetic abnormalities may be related to inflammatory diseases other than FMF. For example, Japanese patients with rheumatoid arthritis are known to have a higher frequency of MEFV mutations. ${ }^{9}$ Also, there is a case report of colchicine-responsive chronic recurrent multifocal osteomyelitis (CRMO) in a 14-year-old Japanese female with these mutations. ${ }^{11}$ These mutations were also seen in higher frequency in patients with systemic onset juvenile idiopathic arthritis (SoJIA) ${ }^{12}$ and Behçet's disease. ${ }^{13-17}$ Further accumulation of clinical data on these mutations will help our understanding of the roles of these mutations.

In conclusion, we saw a case of recurrent serositis consisting with FMF despite the lack of fever. There were multiple MEFV gene mutations, and these may be related to the symptoms. Astute clinical suspicion is important in diagnosing FMF without fever. Further studies will be needed to delineate the precise nature of FMF in Japanese people.

\section{Disclosure}

The authors report no conflicts of interest in this work.

\section{References}

1. Livneh A, Langevitz P, Zemer D, et al. Criteria for the diagnosis of familial Mediterranean fever. Arthritis Rheum. 1997;40(10):1879-1885.

2. Yamazaki K, Yamazaki T, Masumoto J, Suzuki A, Yazaki M, Agematsu K. Familial Mediterranean fever as representative autoinflammatory disease. Rinsho Byori. 2009;57(4):371-381. Japanese. 
3. Tsuchiya-Suzuki A, Yazaki M, Nakamura A, et al. Clinical and genetic features of familial Mediterranean fever in Japan. J Rheumatol. 2009; 36(8):1671-1676.

4. Iwata K, Toma T, Yachie A, et al. Case of recurrent fever with apparent response to antibiotic: Overcoming "CRP dependency". General Medicine. 2011;12(1):29-31.

5. Yamane T, Uchiyama K, Hata D, et al. A Japanese case of familial Mediterranean fever with onset in the fifties. Intern Med. 2006;45(8): 515-517.

6. Tunca M, Akar S, Onen F, et al. Familial Mediterranean fever (FMF) in Turkey: results of a nationwide multicenter study. Medicine (Baltimore). 2005;84(1):1-11.

7. Pras E, Livneh A, Balow JE Jr, et al. Clinical differences between North African and Iraqi Jews with familial Mediterranean fever. Am J Med Genet. 1998;75(2):216-219.

8. Saito M, Nishikomori R, Kambe N. Familial Mediterranean fever: MEFV gene mutations and treatment. Nihon Rinsho Meneki Gakkai Kaishi. 2007;30(2):78-85. Japanese.

9. Migita K, Nakamura T, Maeda Y, et al. MEFV mutations in Japanese rheumatoid arthritis patients. Clin Exp Rheumatol. 2008;26(6): 1091-1094.

10. Ryan JG, Masters SL, Booty MG, et al. Clinical features and functional significance of the P369S/R408Q variant in pyrin, the familial Mediterranean fever protein. Ann Rheum Dis. 2010;69(7):1383-1388.
11. Shimizu M, Tone $\mathrm{Y}$, Toga A, et al. Colchicine-responsive chronic recurrent multifocal osteomyelitis with MEFV mutations: a variant of familial Mediterranean fever? Rheumatology (Oxford). 2010;49(11): 2221-2223.

12. Ayaz NA, Ozen S, Bilginer Y, et al. MEFV mutations in systemic onset juvenile idiopathic arthritis. Rheumatology (Oxford). 2009;48(1): 23-25.

13. Touitou I, Magne X, Molinari N, et al. MEFV mutations in Behçet's disease. Hum Mutat. 2000;16(3):271-272.

14. Ben-Chetrit E, Cohen R, Chajek-Shaul T. Familial mediterranean fever and Behçet's disease - are they associated? J Rheumatol. 2002;29(3): 530-534.

15. Atagunduz P, Ergun T, Direskeneli H. MEFV mutations are increased in Behçet's disease (BD) and are associated with vascular involvement. Clin Exp Rheumatol. 2003;21(4 Suppl 30):S35-S37.

16. Imirzalioglu N, Dursun A, Tastan B, Soysal Y, Yakicier MC. MEFV gene is a probable susceptibility gene for Behçet's disease. Scand J Rheumatol. 2005;34(1):56-58.

17. Ozen S, Bakkaloglu A, Yilmaz E, et al. Mutations in the gene for familial Mediterranean fever: do they predispose to inflammation? J Rheumatol. 2003;30(9):2014-2018.
International Journal of General Medicine

\section{Publish your work in this journal}

The International Journal of General Medicine is an international, peer-reviewed open-access journal that focuses on general and internal medicine, pathogenesis, epidemiology, diagnosis, monitoring and treatment protocols. The journal is characterized by the rapid reporting of reviews, original research and clinical studies across all disease areas.

\section{Dovepress}

A key focus is the elucidation of disease processes and management protocols resulting in improved outcomes for the patient.The manuscript management system is completely online and includes a very quick and fair peer-review system. Visit http://www.dovepress.com/ testimonials.php to read real quotes from published authors. 